\title{
Synthesis and hydrogen-storage performance of interpenetrated MOF-5/MWCNTs hybrid composite with high mesoporosity
}

\author{
Heng Jiang, Yafei Feng, Meng Chen, Yuren Wang* \\ Key Laboratory of Microgravity, Institute of Mechanics, Chinese Academy of Sciences, No. 15 Beisihuanxi Road, \\ Beijing 100190, PR China
}

\section{A R T I C L E I N F O}

Article history:

Received 16 November 2012

Received in revised form

6 March 2013

Accepted 8 March 2013

Available online 6 April 2013

Keywords:

Metal-organic frameworks

Interpenetrated structure

Hydrogen-storage

Multi-walled carbon nanotubes

High mesoporosity

\begin{abstract}
A B S T R A C T
Metal-organic frameworks (MOFs) exhibiting high surface area and tunable pore size own broad application prospects. Compared with existing MOFs, MOF-5 $\left[\mathrm{Zn}_{4} \mathrm{O}(\mathrm{bdc})_{3}\right]$ is a promising hydrogen storage material due to high $\mathrm{H}_{2}$ uptake capacity and thermostability. However, further wider applications of MOF-5 have been limited because atmospheric moisture levels cause MOF-5 instability. MOF-5 and multi-walled carbon nanotubes (MWCNTs) hybrid composite (denoted MOFMC) can enhance stability toward ambient moisture and improve hydrogen storage capacity. In this paper, the MOFMC, which has an interpenetrated structure with high mesoporosity, was synthesized. The MOFMC is denoted as Int-MOFMC-meso. It stored $2.02 \mathrm{wt} \% \mathrm{H}_{2}$ at $77 \mathrm{~K}$ under 1 bar, which is higher than the MOF- 5 with similar structure and the earlier reported MOFMC material. Moreover, the Int-MOFMC-meso can also show more excellent performance of thermostability and moisture stability than the MOF-5 with similar structure.

Copyright $\odot$ 2013, Hydrogen Energy Publications, LLC. Published by Elsevier Ltd. All rights
\end{abstract}

reserved.

\section{Introduction}

In the past decade, metal-organic frameworks (MOFs), which exhibit high surface area, low density and tunable pore size [1-4], have inspired great interest because they can own broad application prospects such as gas storage, sorption, optics and catalysis [5-10]. MOF-5 $\left[\mathrm{Zn}_{4} \mathrm{O}(\mathrm{bdc})_{3}\right.$; bdc $=1,4-$ benzenedicarboxylate], which possesses a zeolite-like framework, has come up with fairly high $\mathrm{H}_{2}$ capacity and the most thermostable [11-14]. Compared with existing MOFs, MOF-5 is a promising hydrogen storage material [15]. The crystal structure and pore texture properties of MOF- 5 giving rise to different adsorption properties are affected by different approaches [16], but measurements show that their XRD peak positions are essentially the same except for the peak intensities [17]. Recent studies showed that the interpenetrated MOF-5 material possesses a higher hydrogen storage capacity than the non-interpenetrated MOF-5 at $77 \mathrm{~K}$ under 1 bar. The performance of MOF-5 is improved by the interpenetrated structure to a certain extent. However, harsh conditions of MOF-5 for sorption or storage $\mathrm{H}_{2}$ and moisture instability are both important challenges for further wider applications. In this respect, a hybrid composite is noticed. The hybrid composite prepared by incorporation of acid-treated multi-walled carbon nanotubes (MWCNTs) into MOF-5 (denoted MOFMC) can exhibit enhanced specific surface area, increased $\mathrm{H}_{2}$ storage capacity, and improved stability in the presence of ambient moisture, compared with MOF-5 [18]. This research

\footnotetext{
* Corresponding author. Tel.: +86 0108254 4091; fax: +86 01082544096.

E-mail address: yurenwang@imech.ac.cn (Y. Wang).

0360-3199/\$ - see front matter Copyright @ 2013, Hydrogen Energy Publications, LLC. Published by Elsevier Ltd. All rights reserved. http://dx.doi.org/10.1016/j.ijhydene.2013.03.044
} 
presents a new approach for enhancing performance of MOFs. Recently, Yang et al. studied the effects of the structural modifications on the $\mathrm{H}_{2}$ storage capacity for different MOF-5s and synthesized the non-mesoporous MOFMC with the interwoven structure [19]. Experiment results showed that the non-mesoporous MOFMC with the interwoven structure increased $\mathrm{H}_{2}$ storage capacity compared to the conventional MOFMC. However, the peak at $8.4^{\circ}$ in the XRD patterns implies partial collapse of this interwoven MOFMC without mesopores $[13,18,20]$.

Generally speaking, construction of MOF- 5 with the structure of homogeneous micropore is commonly concerned. Recent investigation implied that the combination of micropores and mesopores was favorable for hydrogen storage of MOF-5 [21,22]. In this study, we synthesized the new MOFMC material denoted as Int-MOFMC-meso, which has interpenetrated structure with high mesoporosity. It can store $2.02 \mathrm{wt} \%$ by volumetric method hydrogen at $77 \mathrm{~K}$ under 1 bar, which is higher than the amount stored $1.52 \mathrm{wt} \%$ by the conventional MOFMC. Moreover, the Int-MOFMC-meso also shows high thermostability and stability toward ambient moisture compared with the MOF-5 with similar structure.

\section{Experimental}

\subsection{Reagents and chemicals}

Zinc nitrate hexahydrate $\left(\mathrm{Zn}\left(\mathrm{NO}_{3}\right)_{2} \cdot 6 \mathrm{H}_{2} \mathrm{O}\right)$ (XiLong Chemical Co., Ltd.), terephthalic acid (Sinopharm Chemical Reagent Co., Ltd.), N,N'-dimethylformamide (DMF) (XiLong Chemical Co., Ltd.), Triethylamine (TEA) (XiLong Chemical Co., Ltd.), Multiwalled carbon nanotubes (MWCNTs) (>95\%, diameter: from $50 \mathrm{~nm}$ to $100 \mathrm{~nm}$, Nachen S\&T Ltd.), 4A-type molecular sieve (Sinopharm Chemical Reagent Co., Ltd.), and anhydrous chloroform (Beijing Chemical Plant) were used without further purification.

\subsection{Preparation of functionalized MWCNTs}

MWCNTs were functionalized by acid treatment in (1:3) $\mathrm{HNO}_{3} /$ $\mathrm{H}_{2} \mathrm{SO}_{4}$ mixture, followed by ultrasonication for $4 \mathrm{~h}$ at $333 \mathrm{~K}$. The reaction mixture was diluted with ultrapure water until the suspension $\mathrm{pH}$ was nearly neutral. Then the suspension was centrifugated. The MWCNTs were collected and dried at $373 \mathrm{~K}$.

\subsection{Preparation of the interpenetrated MOF-5 with mesopores}

$\mathrm{Zn}\left(\mathrm{NO}_{3}\right)_{2} \cdot 6 \mathrm{H}_{2} \mathrm{O}(5.60 \mathrm{mmol})$ and terephthalic acid $\left(\mathrm{H}_{2} \mathrm{BDC}\right.$, $2.12 \mathrm{mmol}$ ) were dissolved in $50 \mathrm{~mL}$ of dehydrated DMF solvent. Triethylamine (TEA, $550 \mu \mathrm{L}$ ) was added into the mixture and white products were filtrated immediately. The transparent solution quickly transferred to a $100 \mathrm{~mL}$ glass vial and sealed. The glass vial was then heated to $378 \mathrm{~K}$ for $24 \mathrm{~h}$. After the reaction, the vial was cooled down to the room temperature naturally. The powders were collected and washed thoroughly with DMF. After that, the powders were immersed in chloroform for 7 days. During the process, the solvent was decanted and replenished every two days. The prepared MOF-5 powders were dried under vacuum at $428 \mathrm{~K}$ for $24 \mathrm{~h}$.

\subsection{Preparation of the Int-MOFMC-meso}

$\mathrm{Zn}\left(\mathrm{NO}_{3}\right)_{2} \cdot 6 \mathrm{H}_{2} \mathrm{O}(5.60 \mathrm{mmol})$ and terephthalic acid $\left(\mathrm{H}_{2} \mathrm{BDC}\right.$, $2.12 \mathrm{mmol}$ ) were dissolved in $50 \mathrm{~mL}$ of dehydrated DMF solvent. Triethylamine (TEA, $550 \mu \mathrm{L}$ ) was added into the mixture and white products were filtrated immediately. Then $50 \mathrm{mg}$ acid-treated MWCNTs were added into the transparent solution and stirred for $24 \mathrm{~h}$. The black solution was quickly transferred to a $100 \mathrm{~mL}$ glass vial and sealed. The following steps are the same as the steps in the processes of MOF-5.

\subsection{Characterization}

The morphologies of sample were obtained using a HITACHIS4300 scanning electron microscope (SEM) and a Nikon DIAPHOT 300 optical microscope. X-ray diffraction (XRD) pattern of sample was obtained on a Rigaku Ultima IV X-ray diffractometer with a $\mathrm{Cu} \mathrm{K} \alpha 1$ radiation source $(k=1.54056 \AA)$ operated at $40 \mathrm{kV}$ and $40 \mathrm{~mA}$ at a scanning step of $0.01^{\circ}$ in the $2 \theta$ range $5^{\circ}-40^{\circ}$. Thermal behavior of the sample was examined using TA TGA Q5000IR under $\mathrm{N}_{2}$ stream with a heating rate of $5{ }^{\circ} \mathrm{C} / \mathrm{min}$ from room temperature to $600{ }^{\circ} \mathrm{C}$. Nitrogen adsorption/desorption analysis was measured using a Micromeritics ASAP 2020M apparatus. Before the measurement, the samples were heated at $403 \mathrm{~K}$ for $10 \mathrm{~h}$. The hydrogen adsorption measurement (volumetric method) was measured at $77 \mathrm{~K}$ under 1 bar pressure using the same gas sorption apparatus.

\section{Results and discussion}

Fig. 1 shows the SEM and optical micrographs of the asprepared MOF-5 and the Int-MOFMC-meso. Fig. 1(a) exhibits the optical image of the as-prepared MOF-5. Fig. 1(b) shows the optical micrographs of the as-prepared Int-MOFMC-meso. Its morphology is characterized by well-defined cubic crystals 20-150 $\mu \mathrm{m}$ in width. The as-prepared black Int-MOFMC-meso cubic crystal indicates that large numbers of CNTs were incorporated into MOF-5. The as-prepared MOF-5 is observed by SEM image, as shown in Fig. 1(c). Fig. 1(d) displays SEM micrographs of the as-prepared Int-MOFMC-meso. The interpenetrated structure defined by Han et al. is that the frameworks are maximally displaced from each other by shifting the second framework exactly one half of the pore size in the $x, y$, and $z$ directions [23]. According to the definition, the morphologies of the as-prepared MOF-5 and the Int-MOFMCmeso might be result from the interpenetration.

$\mathrm{XRD}$ investigation for the as-prepared MOF-5 and the IntMOFMC-meso crystalline powder are reported in Fig. 2. The intensity of peaks and their sharpness imply high crystallinity of two samples, as shown in Fig. 1. The XRD pattern of the IntMOFMC-meso is similar to the as-prepared MOF-5 crystalline phase, thus confirming that MWCNTs incorporation did not disturb or destroy the interpenetrated MOF-5 crystal structure [18]. The diffraction peaks from the MWCNTs supporters cannot be observed, because MWCNTs was swamped by highintensity MOF-5 peaks. According to Chen et al., the 

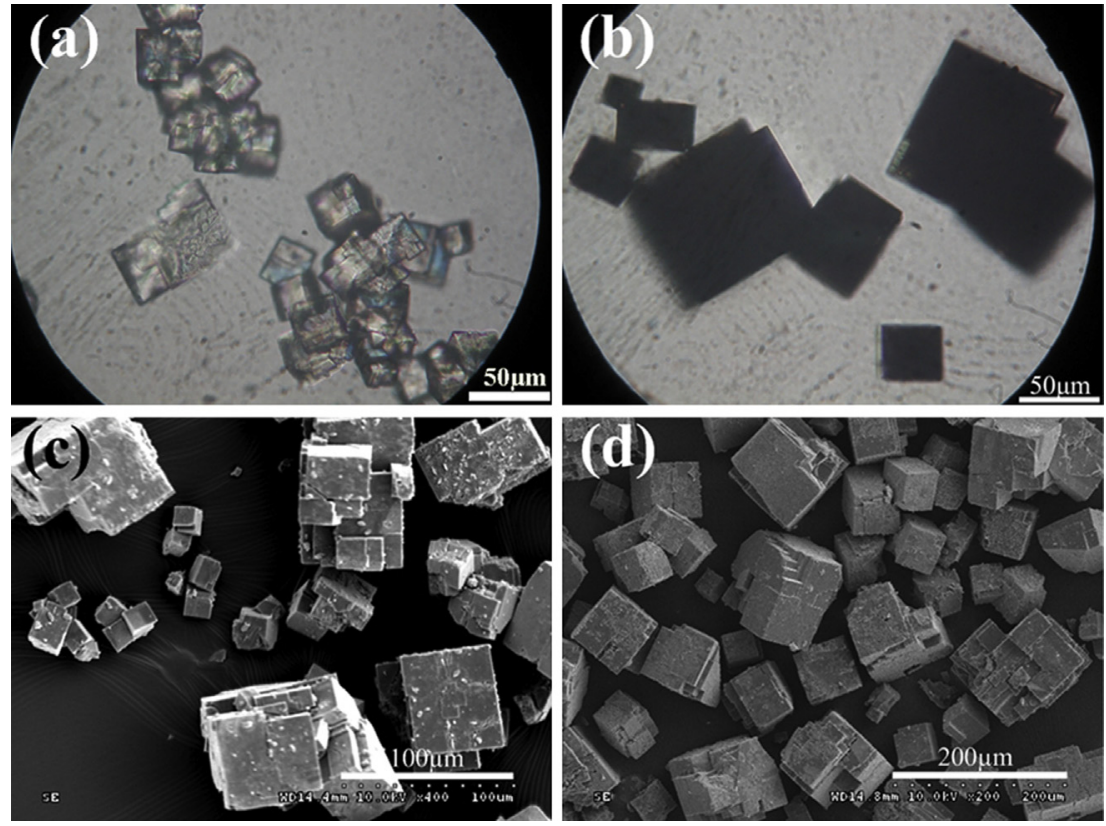

Fig. 1 - Optical photos of (a) the interpenetrated MOF-5 with mesopores and (b) the Int-MOFMC-meso samples, SEM images of (c) the interpenetrated MOF-5 with mesopores and (d) the Int-MOFMC-meso samples.

intensities of the XRD peaks of MOF-5 are crucial to estimate the interpenetrated structure, especially the intensities of the peaks at $6.8^{\circ}, 9.7^{\circ}$ and $13.6^{\circ}$. The high intensity ratio of the XRD peak at $13.8^{\circ}$ to the peak at $6.8^{\circ}$ (referred to as the $R_{2}$ value) can be used to predict the interpenetrated structure, especially when the $R_{1}$ value (the ratio of the intensity of the peak at $9.7^{\circ}$ to that at $6.8^{\circ}$ ) is low [17]. The reference [17] also shows that different methods of preparation result in various $R_{2}$ value. The MOF-5 and MOFMC reported in this paper possesses high $R_{2}$ and relative low $R_{1}$ which means that they have interpenetrated structure.
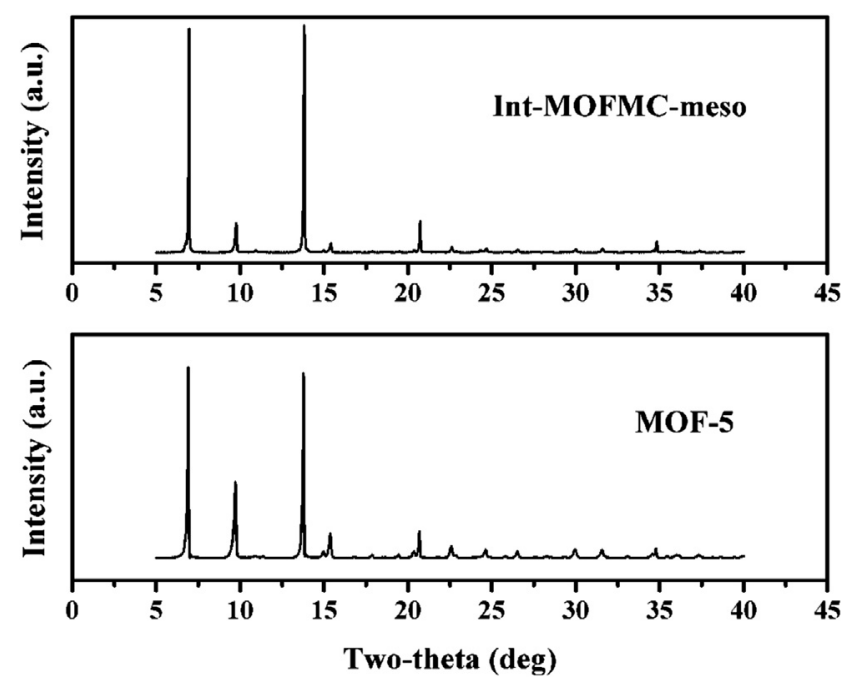

Fig. 2 - Powder X-ray diffraction patterns of the interpenetrated MOF-5 with mesopores and the Int-MOFMC-meso.
Fig. 3 shows that moisture stability tests on the interpenetrated MOF-5 with mesopores and the Int-MOFMC-meso were achieved in humid air. Fig. 3(a) confirms that XRD peak of the Int-MOFMC-meso did not change even after 6 days in humid air. Fig. 3(b) shows that a new XRD peak of the asprepared MOF-5 appeared at around $8.4^{\circ}$, when it exposed to humid air for 2 days. The presence of the peak at $8.4^{\circ}$ implies partial collapse of the structure of the as-prepared MOF-5 $[13,18,20]$. The peak at around $8.4^{\circ}$ relative intensity increased and some new XRD peaks appeared with exposure time, indicating acceleration of decomposition. Therefore, the Int-
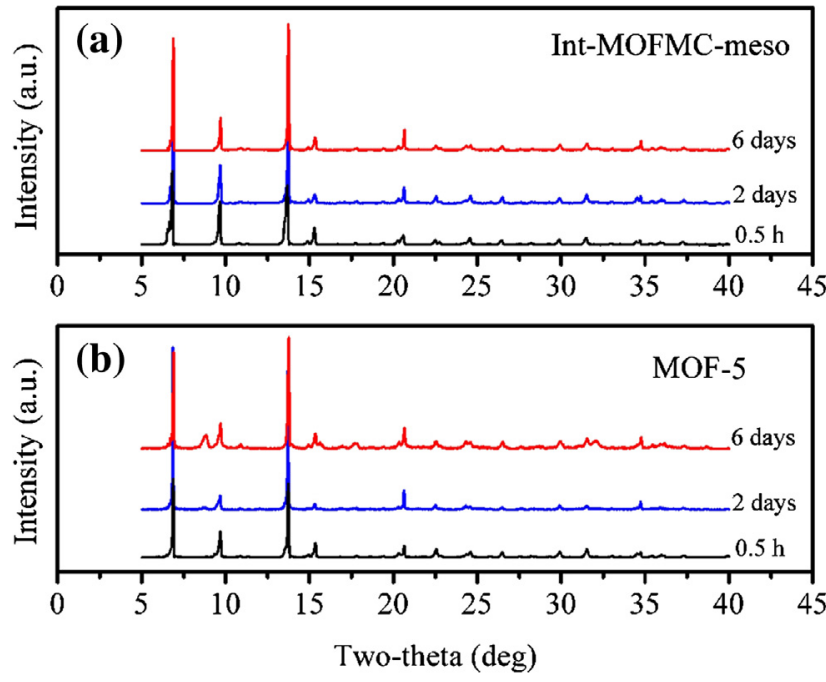

Fig. 3 - Powder X-ray diffraction patterns for (a) the IntMOFMC-meso and (b) the interpenetrated MOF-5 with mesopores exposed to static humid air conditions for $0.5 \mathrm{~h}$, 2 days and 6 days. 
MOFMC-meso possesses moisture stability of under ambient conditions due to incorporation of MWCNTs into MOF-5 crystals [18].

The TGA curves of the interpenetrated MOF- 5 with mesopores and the Int-MOFMC-meso are reported in Fig. 4. Because the interpenetrated MOF-5 with mesopores and the IntMOFMC-meso have the similar decomposition process, the Int-MOFMC-meso as an example shows decomposition process. Firstly, the Int-MOFMC-meso exhibits a 1.9\% weight loss below $80{ }^{\circ} \mathrm{C}$, which is assigned to desorption of surface adsorbed water. Then no weight loss of DMF solvent is observed in the range $80-240{ }^{\circ} \mathrm{C}$, owing to no residual DMF solvent in the pores after activation. Generally, zinc hydroxide $\left(\mathrm{Zn}(\mathrm{OH})_{2}\right)$ can be dehydrated at $125^{\circ} \mathrm{C}$ [17], the TGA confirms that zinc species does not exist in Int-MOFMC-meso. Finally, a weight loss of about $51 \%$ takes place and corresponds to the structural decomposition. MWCNTs in the Int-MOFMC-meso enhance thermal stability compared to the MOF-5 with similar structure, as confirmed by a rise in decomposition temperature from 470 to $495^{\circ} \mathrm{C}$.

Fig. 5 shows $\mathrm{N}_{2}$ adsorption/desorption isotherm at $77 \mathrm{~K}$ and 1 bar and the pore size distributions (PSDs) of the Int-MOFMCmeso. Fig. 5(a) indicates a sharply increasing step before $0.01 \mathrm{P} / \mathrm{P}_{0}$ and then a quick saturation step at low pressure, and the type H3 hysteresis loop at higher relative pressure [24]. The results imply that micro- and mesopores coexist in the Int-MOFMC-meso, and the type of hysteresis loop indicates the existence of narrow slit-like intercrystalline void [25-28]. Fig. 5(b) and (c) exhibit size distributions of micro- and mesopores. Generally, the pore size can be decreased by the interpenetrated structure because the micropore size of the non-interpenetrated MOF-5 is larger than $1 \mathrm{~nm}$ [29]. Fig. 5(b) shows that the micropores mainly focus on the range of $0.52-0.8 \mathrm{~nm}$, which indicates interpenetrated structure in the Int-MOFMC-meso. Fig. 5(c) confirms that the PSDs of mesopore are wide. The Langmuir surface area of the Int-MOFMCmeso is about $1035 \mathrm{~m}^{2} \mathrm{~g}^{-1}$, which is low to the reported $3550 \mathrm{~m}^{2} \mathrm{~g}^{-1}$ of MOFMC by Yang. The Brunauer-Emmett-Teller (BET) surface area of the Int-MOFMC-meso is about

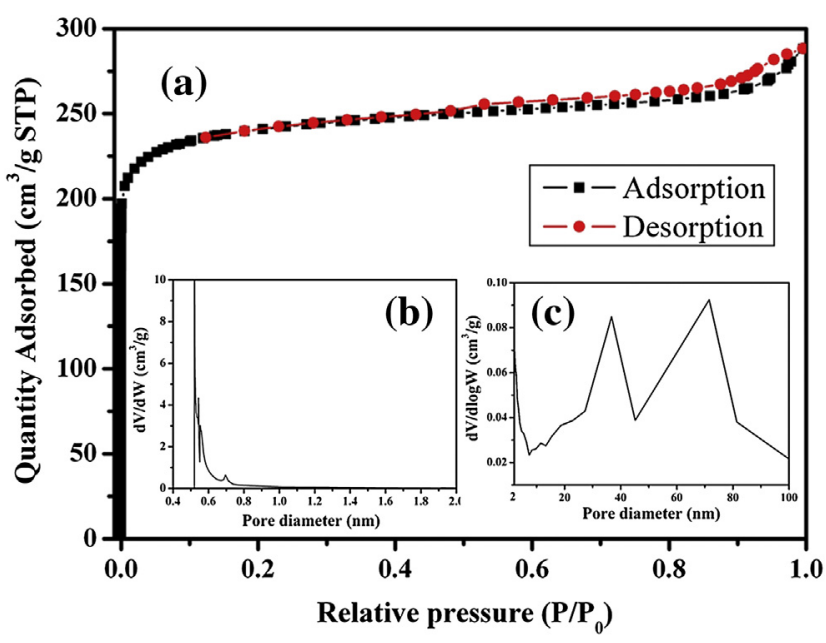

Fig. 5 - (a) $\mathrm{N}_{2}$ adsorption and desorption isotherms of the Int-MOFMC-meso. (b) The micropores distribution of the Int-MOFMC-meso calculated by $\mathrm{H}-\mathrm{K}$ method. (c) The mesopores distribution of the Int-MOFMC-meso calculated by BJH method.

$805 \mathrm{~m}^{2} \mathrm{~g}^{-1}$, which is low to the reported $2900 \mathrm{~m}^{2} \mathrm{~g}^{-1}$ of MOFMC by Yang. Generally, the low SSA of MOF-5 material is either due to pore filled by zinc species or solvent, the interpenetrated structure [30], or the mesopores in the material [15]. The low $R_{1}$ of XRD in Fig. 2 can imply pores of the IntMOFMC-meso without or with small amounts trapped zinc species or solvent [30]. Fig. 4 further confirms there is no zinc species and solvent in pores of the Int-MOFMC-meso. Therefore, the main reason resulting in low SSA of the Int-MOFMCmeso is not pore-filling but interpenetrated structure and high mesopores.

Hydrogen adsorption contrast curves between the interpenetrated MOF-5 with mesopores and the Int-MOFMC-meso samples at $77 \mathrm{~K}$ under $0-1$ bar are given in Fig. 6 . The result

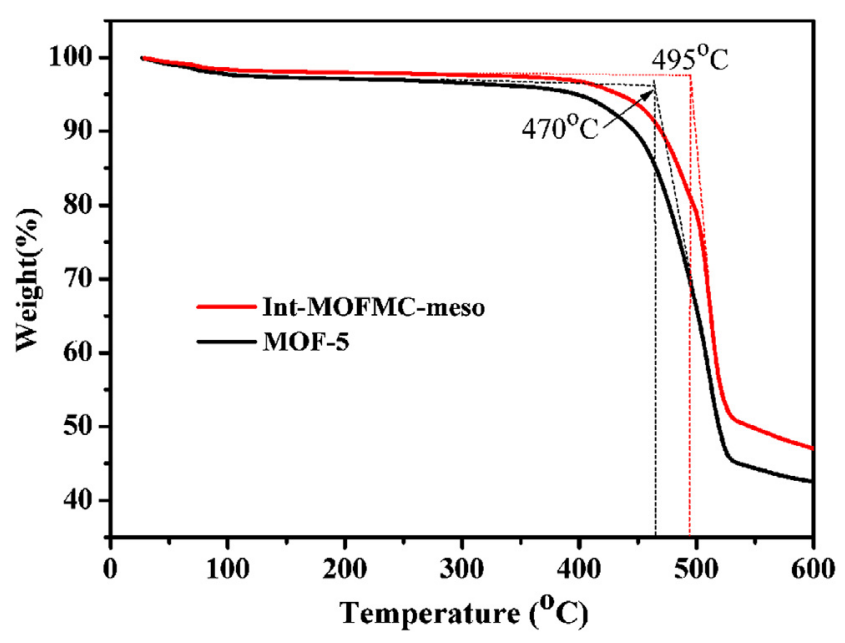

Fig. 4 - TGA curves of the interpenetrated MOF-5 with mesopores and the Int-MOFMC-meso samples.

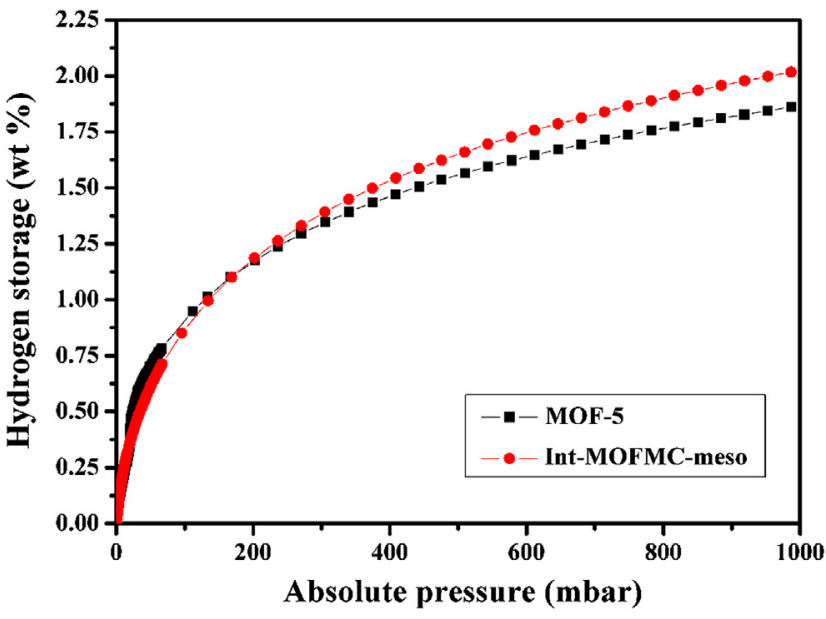

Fig. 6 - Hydrogen adsorption contrast curves between the interpenetrated MOF-5 with mesopores and the IntMOFMC-meso samples at $77 \mathrm{~K}$ under 1 bar. 
shows that the adsorption reached $2.02 \mathrm{wt} \%$ at $77 \mathrm{~K}$ under 1 bar of the Int-MOFMC-meso, which is greater than the MOF- 5 with similar structure and the earlier reported $1.52 \mathrm{wt} \%$ of the conventional MOFMC without mesopores by Yang under the same conditions [18]. It is well known that the interpenetrated structure is more effective in hydrogen uptake than the noninterpenetrated MOFs [31,32]. Fig. 5 shows that micropores 0.52-0.8 $\mathrm{nm}$ in diameter formed from the interpenetrated structure have a greater distribution in the Int-MOFMC-meso, which is favorable for hydrogen storage. Moreover, MWCNTs play an important role in enhancing the hydrogen uptake for MOF-5 [18]. In addition, mesopores do not destroy the hydrogen uptake of as-prepared Int-MOFMC-meso, which is more likely due to the strong hydrogen physisorption resulting from the unsaturated metal sites on the surface of intercrystalline mesopores. This can also be invoked to imply the presence of hydrogen storage peak over $2 \mathrm{wt} \%$ in the hydrogen adsorption spectrum.

\section{Conclusions}

In conclusion, we have synthesized the new MOFMC material, which possesses interpenetrated structure and hierarchical nanopores. Compared to the MOF- 5 with similar structure and the reported MOFMC material, our sample includes high mesoporosity and hydrogen storage $(2.02 \mathrm{wt} \%$ at $77 \mathrm{~K}$ and 1 bar) was considerably improved. The Int-MOFMC-meso exhibits better thermostability and moisture stability than the MOF-5 with similar structure.

\section{Acknowledgements}

The authors wish to acknowledge the support from the National Natural Science Foundation of China (Nos. 11202211 and 11272315).

\section{R E F E R E N C E S}

[1] Chae HK, Siberio-Pérez DY, Kim J, Go YB, Eddaoudi M, Matzger AJ, et al. A route to high surface area, porosity and inclusion of large molecules in crystals. Nature 2004;427:523-7.

[2] Rosi NL, Eckert J, Eddaoudi M, Vodak DT, Kim J, O'Keeffe M, et al. Hydrogen storage in microporous metal-organic frameworks. Science 2003;300:1127-9.

[3] Rowsell JLC, Eckert J, Yaghi OM. Characterization of $\mathrm{H}_{2}$ binding sites in prototypical metal-organic frameworks by inelastic neutron scattering. J Am Chem Soc 2005;127:14904-10.

[4] Prasanth KP, Rallapalli P, Raj MC, Bajaj HC, Jasra RV. Enhanced hydrogen sorption in single walled carbon nanotube incorporated MIL-101 composite metal-organic framework. Int J Hydrogen Energ 2011;36:7594-601.

[5] Sudik AC, Millward AR, Ockwig NW, Cote AP, Kim J, Yaghi OM. Design, synthesis, structure, and gas $\left(\mathrm{N}_{2}, \mathrm{Ar}, \mathrm{CO}_{2}\right.$, $\mathrm{CH}_{4}$, and $\mathrm{H}_{2}$ ) sorption properties of porous metal-organic tetrahedral and heterocuboidal polyhedra. J Am Chem Soc 2005;127:7110-8.
[6] Assfour B, Seifert G. Hydrogen storage in 1D nanotube-like channels metal-organic frameworks: effects of free volume and heat of adsorption on hydrogen uptake. Int J Hydrogen Energ 2009;34:8135-43.

[7] Bourrelly S, Llewellyn PL, Serre C, Millange F, Loiseau T, Férey G. Different adsorption behaviors of methane and carbon dioxide in the isotypic nanoporous metal terephthalates MIL-53 and MIL-47. J Am Chem Soc 2005;127:13519-21.

[8] Seo JS, Whang D, Lee H, Jun SI, Oh J, Jeon YJ, et al. A homochiral metal-organic porous material for enantioselective separation and catalysis. Nature 2000;404:982-6.

[9] Haneda T, Kawano M, Kojima T, Fujita M. Thermo-to-photoswitching of the chromic behavior of salicylideneanilines by inclusion in a porous coordination network. Angew Chem Int Ed 2007;46:6643-5.

[10] Wong-Foy AG, Matzger AJ, Yaghi OM. Exceptional $\mathrm{H}_{2}$ saturation uptake in microporous metal-organic frameworks. J Am Chem Soc 2006;128:3494-5.

[11] Saha D, Deng S. Synthesis, characterization and hydrogen adsorption in mixed crystals of MOF-5 and MOF-177. Int J Hydrogen Energ 2009;34:2670-8.

[12] Purewal JJ, Liu D, Yang J, Sudik A, Siegel DJ, Maurer S, et al. Increased volumetric hydrogen uptake of MOF- 5 by powder densification. Int J Hydrogen Energ 2012;37:2723-7.

[13] Kaye SS, Dailly A, Yaghi OM, Long JR. Impact of preparation and handling on the hydrogen storage properties of $\mathrm{Zn}_{4} \mathrm{O}$ (1,4-benzenedicarboxylate) ${ }_{3}$ (MOF-5). J Am Chem Soc 2007;129:14176-7.

[14] Zhou W, Wu H, Hartman MR, Yildirim T. Hydrogen and methane adsorption in metal-organic frameworks: a highpressure volumetric study. J Phys Chem C 2007;111:16131-7.

[15] Tsao CS, Yu MS, Chung TY, Wu HC, Wang CY, Chang KS, et al. Characterization of pore structure in metal-organic framework by small-angle X-ray scattering. J Am Chem Soc 2007;129:15997-6004.

[16] Huang L, Wang H, Chen J, Wang Z, Sun J, Zhao D, et al. Synthesis, morphology control, and properties of porous metal-organic coordination polymers. Microporous Mesoporous Mater 2003;58:105-14.

[17] Chen B, Wang X, Zhang Q Xi X, Cai J, Qi H, et al. Synthesis and characterization of the interpenetrated MOF-5. J Mater Chem 2010;20:3758-67.

[18] Yang SJ, Choi JY, Chae HK, Cho JH, Nahm KS, Park CR. Preparation and enhanced hydrostability and hydrogen storage capacity of CNT@MOF-5 hybrid composite. Chem Mater 2009;21:1893-7.

[19] Yang SJ, Jung H, Kim T, Im JH, Park CR. Effects of structural modifications on the hydrogen storage capacity of MOF-5. Int J Hydrogen Energ 2012;37:5777-83.

[20] Greathouse JA, Allendorf MD. The interaction of water with MOF-5 simulated by molecular dynamics. J Am Chem Soc 2006;128:10678-9.

[21] Xin Z, Bai J, Pan Y, Zaworotko MJ. Synthesis and enhanced $\mathrm{H}_{2}$ adsorption properties of a mesoporous nanocrystal of MOF-5: controlling nano-/mesostructures of MOFs to improve their $\mathrm{H}_{2}$ heat of adsorption. Chem Eur J 2010;16:13049-52.

[22] Du H, Bai J, Zuo C, Xin Z, Hu J. A hierarchical suprananostructure of HKUST-1 featuring enhanced $\mathrm{H}_{2}$ adsorption enthalpy and higher mesoporosity. CrystEngComm 2011;13:3314-6.

[23] Han SS, Mendoza-Cortés JL, Goddard III WA. Recent advances on simulation and theory of hydrogen storage in metal-organic frameworks and covalent organic frameworks. Chem Soc Rev 2009;38:1460-76. 
[24] Sing KSW, Williams RT. Physisorption hysteresis loops and the characterization of nanoporous materials. Adsorpt Sci Technol 2004;22:773-82.

[25] Vishnyakov A, Ravikovitch PI, Neimark AV, Bülow M, Wang QM. Nanopore structure and sorption properties of Cu-BTC metal-organic framework. Nano Lett 2003;3:713-8.

[26] Lee JY, Li J, Jagiello J. Gas sorption properties of microporous metal organic frameworks. J Solid State Chem 2005;178:2527-32.

[27] Luzan SM, Talyzin AV. Hydrogen adsorption in Pt catalyst/ MOF-5 materials. Microporous Mesoporous Mater 2010;135:201-5.

[28] Lee SY, Park SJ. Effect of platinum doping of activated carbon on hydrogen storage behaviors of metal-organic frameworks-5. Int J Hydrogen Energ 2011;36:8381-7.
[29] Yaghi OM, O’Keeffe M, Ockwig NW, Chae HK, Eddaoudi M, Kim J. Reticular synthesis and the design of new materials. Nature 2003;423:705-14.

[30] Hafizovic J, Bjørgen M, Olsbye U, Dietzel PDC, Bordiga S, Prestipino $\mathrm{C}$, et al. The inconsistency in adsorption properties and powder XRD data of MOF- 5 is rationalized by framework interpenetration and the presence of organic and inorganic species in the nanocavities. J Am Chem Soc 2007;129:3612-20.

[31] Yushin G, Dash R, Jagiello J, Fischer JE, Gogotsi Y. Carbidederived carbons: effect of pore size on hydrogen uptake and heat of adsorption. Adv Funct Mater 2006;16:2288-93.

[32] Cabria I, López MJ, Alonso JA. The optimum average nanopore size for hydrogen storage in carbon nanoporous materials. Carbon 2007;45:2649-58. 